\title{
La Mona Lisa: un compendio de Medicina Interna
}

\author{
A. MARTÍNEZ GARCÍA
}

THE MONA LISA: A COMPENDIUM OF INTERNAL MEDICINE

\begin{abstract}
RESUMEN
A través de la historia de la pintura muchos artistas han retratado en sus obras signos de diferentes patologías. En algunos casos, dichas enfermedades son fácilmente reconocibles, aunque en otros se necesita un ojo clínico bien entrenado para alcanzar el diagnóstico. De entre las muchas obras que han atraído el interés médico, ninguna comparable con el retrato más famoso y enigmático pintado por Leonardo da Vinci, la Mona Lisa.
\end{abstract}

PALABRAS CLAVE: Arte. Historia. Mona Lisa. Enfermedad.

\begin{abstract}
Through the history of art many artists in their works have portrayed different signs of pathologies. In some cases, the illnesses are easily recognised, although in others a well trained specialist is necessary to reach the diagnosis. Within the numerous works of art that have attracted medical interest, there is none comparable to the most famous and enigmatic portrait painted by Leonardo da Vinci, the Mona Lisa.
\end{abstract}

Martínez García A. La Mona Lisa: un compendio de Medicina Interna. An Med Interna (Madrid) 2006; $139-141$.

La historia de la pintura, y en particular del retrato, aparece como una de las expresiones de la creatividad humana donde de un modo más natural se conjugan arte y patología. La gran capacidad de observación que caracteriza al artista ha hecho posible en no pocas ocasiones que, inadvertida o conscientemente, queden reflejadas en sus obras las manifestaciones físicas y psíquicas de múltiples y variadas enfermedades. Estos signos clínicos, en algunos casos, resaltan de forma tan evidente como en muchos de los retratos velazqueños; por el contrario, en otras obras sólo aparecen perceptibles a los ojos del especialista o del profesional avezado, siendo estas últimas, no cabe ninguna duda, las que plantean el reto más estimulante y las preferidas para sentar en la consulta. En el intento de hacer frente a ese mudo bis a bis con el personaje retratado, de la clásica propedéutica clínica sólo podemos contar con la inspección y el interrogatorio. Para la primera se necesitan amplios conocimientos médicos, imaginación y, sobre todo, dominar el difícil arte de mirar y ver, en suma, tener un muy perspicaz ojo clínico. Por su parte, el interrogatorio hay que dirigirlo necesariamente sobre los documentos y fuentes bibliográficas que en la historia del arte se refieren a la obra en cuestión. Aunque en la historia del retrato existen múltiples obras que han atraído el interés diagnóstico del médico aficionado a la pintura, ninguna comparable con la enigmática e irresistible Gioconda. Un atractivo quizá derivado, como señala Gombrich (1), de la impresión de viva realidad que transmite la sonrisa y la mirada penetrante de una joven que parece observarnos y pensar por ella misma.

En su silencioso deambular de consulta en consulta, la historia clínica de la Gioconda ha ido acumulando diagnósticos de casi todas las especialidades médicas. En 1959 el Dr. Keele (2), experto en la obra científica de Leonardo, diagnosticó el embarazo de la modelo al identificar en cara, cuello y manos algunos de los cambios externos originados por las típicas alteraciones hormonales de la gestación, amén de la que el autor considera postura típica: los brazos cruzados sobre el regazo; opinión recientemente compartida por el Dr. Nulland (3). Entre ambos, el Dr. Marañón (4) detectó una insuficiencia ovárica, hablando de mano hipogenital con aspecto de impregnación vasculolinfática, sensación de frialdad y que dejarían fóvea al apretarlas. En relación con el embarazo y el mayor riesgo en estos casos de parálisis facial, en 1989 el Dr. Adour (5) identificó una parálisis de Bell parcialmente recuperada, aunque con las secuelas de una leve contractura muscular facial, evidente en la comisura bucal y ceja del lado izquierdo, y una desagradable sincinesia secundaria que provocaría continuos movimientos involuntarios faciales asociados a otros voluntarios o al simple parpadeo, lo que habría hecho imposible concluir la obra y obligado a Leonardo a una indefinición de rasgos. En este mismo sentido se ha manifestado Mantkelow (6), mientras que el prof. Schutzenberg (7) se decanta por una contracción levemente tetanizada de los risorios de Santorini y el gran cigomático, bien de causa congénita o adquirida. Por su parte, el Dr. Borkowski (8) descubre

Trabajo aceptado: 21 de diciembre de 2005

Correspondencia: A. Martínez García. C/ Aniceto Marinas, 2 - 5 A. 28008. Madrid. 
bajo el labio inferior de la joven una lesión cicatricial posiblemente secundaria a un traumatismo bucal con pérdida de piezas dentales, lo que ocasionaría la ausencia de sonrisa franca y la presencia de su característica boca. A este respecto, el Dr. Gargantilla (9) diagnostica un bruxismo por estrés, lo que obligaba a la modelo a encajar bien ambos maxilares para evitarlo, aunque también apunta la posibilidad de que el gesto se deba a una falta de piezas dentales como consecuencia de las frecuentes piorreas de la época. Por otro lado, y en relación con ese peculiar esbozo de sonrisa, se ha lanzado la hipótesis de un ennegrecimiento de las piezas dentales por el tratamiento con mercuriales de la sífilis (10) que padecería la modelo. También comparten el bruxismo por estrés, bien del continuo posar o por el reciente fallecimiento de un hijo, el prof. Miguel Lucas (11) y el Dr. Filippo Surano (12). La esclerodermia (13), de mayor incidencia femenina, es otra patología identificada según el aspecto tenso y adherido a planos profundos de la piel de la cara, cuello, pecho y manos, junto con finos labios y un cierto fruncimiento de la boca. El Dr. Daudén Sala (14), dermatólogo, deteniéndose sobre todo en la ausencia de pelo en cejas y pestañas, habla de un defluvium capillorum, una alopecia universal debida a un estrés emocional mantenido, por lo que debemos suponer que la cabellera de la modelo sería una cuidada peluca. El Dr. Dequeker (15), en relación con un posible xantelasma situado en la proximidad del lagrimal izquierdo y un lipoma de unos tres centímetros de largo en el dorso de la mano derecha por debajo del dedo índice, diagnostica una hiperlipemia o hipercolesterolemia familiar tan severa como para haberle ocasionado la muerte con sólo treinta y siete años. Aunque este autor no detecta la presencia de arco corneal, el especialista japonés, Dr. Nakamuro (16), habla de coloración débilmente amarillenta de la conjuntiva del ojo izquierdo por consumo excesivo de grasas, pero esta heterocromía del iris es interpretada por el Dr. Rodríguez Cabezas (17) como una iridociclitis heterocrómica de Fuchs, donde se asocian la citada heterocromía, uveítis y cataratas. En cuanto al lipoma antes citado, el Dr. Santiago Tamames (18) amplía las posibilidades de dicha lesión nodular a un fibroma, un lipofibroma o simplemente a una elevación fisiológica de la eminencia tenar por la característica disposición de las manos. En relación con esta postura, donde la mano derecha aparece como sujetando a la izquierda, dando la impresión de aferrarse al brazo de la butaca, se ha interpretado (19) como el intento por controlar un temblor de tipo parkinsoniano o también como una siringomielia con atrofia de Aran-Duchene unilateral de esta mano izquierda, de aspecto en garra y con marcada flexión de los dedos índice, medio y anular por retracción de la aponeurosis palmar. Tomando como punto de partida su expresión facial y lo que considera un desplazamiento asimétrico de la sonrisa al lado izquierdo, el Dr. Lay-Son (20) habla de un tic distónico perioral, que sumado a la ausencia de cejas por una posible tricotilomanía, una dificultad de la modelo para mantener la atención y la concentración, o sea, una inquietud motriz, esto último apoyado en el manido relato vasariano de los músicos, cantantes y bufones contratados por Leonardo para entretener y hacer sonreír a la modelo, diagnostica un síndrome de Gilles de la Tourette. La seudosonrisa, como la describe el Dr. Pastore (21), sería en su opinión el gesto forzado por la disnea de una mujer con un cuadro de dificultad respiratoria, un estado asmático que comparte el Dr. Schiarelli (22) y al que se añade cierto estado depresivo. Esbozo de sonrisa que Freeman (23) identifica como típica de la sordera, el prof. Royo-Villanova (24) de ligeramente achispada o etílica, mientras otros (25) ven la sonrisa vacía de la estúpida felicidad presente en la oligofrenia o la debilidad mental.

Tras los múltiples ejercicios de observación, el resultado se manifiesta como un auténtico compendio de medicina interna. Y aunque en nuestra profesión estamos acostumbrados a las inverosimilitudes, parece bastante improbable la concurrencia simultánea de tal cúmulo de patologías en la aparentemente joven que posó para Leonardo. Sin embargo, aún contamos con el recurso del interrogatorio para iluminar y averiguar, hasta donde sea posible, la identidad e historia personal de tan famosa modelo.

Si numerosas son las patologías diagnosticadas, las identidades propuestas para el enigmático retrato que pintó Leonardo hacia 1503-1506, según lo data el Louvre, no se quedan atrás. Tenemos a Caterina Sforza (26) (1463-1509), duquesa de Forli e Imola; a una prima de Caterina, Isabella de Aragón (27) (1470-1524), duquesa de Milán; a la napolitana Isabella Gualanda (28), nacida en 1491 y favorita del duque Giulano de Medicis; a Costanza D'Avalos (29), duquesa de Francavilla e Ischia, nacida en 1460 o a Isabelle D'Este (30) (14741539), esposa de Francisco Gonzaga, marqués de Mantua. Sigmund Freud (31), en la obra que dedicó a la infancia de Leonardo da Vinci, señala a la madre del pintor, Caterina de Anchiano, como la modelo del cuadro. Incluso se ha querido ver un sorprendente autorretrato del propio Leonardo (32). Pero la identidad más verosímil de la famosa modelo, según Zöllner (33), sería la de una joven florentina, Lisa Gherardini, nacida en 1479 en el seno de una familia de la pequeña nobleza rural cercana a Florencia y que contrajo matrimonio, en marzo de 1495, con el acaudalado comerciante en seda y viudo por segunda vez, Francesco di Bartolomeo di Zanobi del Giocondo, nacido en 1460. Coincidiendo con la adquisición de una nueva casa y el reciente nacimiento de su tercer hijo, Andrea, en diciembre de 1502, Francesco del Giocondo, que conocía y mantenía relaciones profesionales con el notario Ser Piero da Vinci, padre de Leonardo, habría encargado el retrato de su esposa. Leonardo comienza el retrato en 1503, es decir, cuando Lisa contaba veinticuatro años, edad difícilmente compatible con una historia clínica tan abigarrada como se ha descrito. Pero por si esto fuera poco, según los datos aportados por Zöllner (34), en 1551 todavía se tienen noticias de Lisa del Giocondo, viuda ya desde 1539, lo que argumenta aún más en contra de tal cúmulo de patologías, o es que quizá tengamos ante nuestros ojos el más sorprendente paradigma del clásico refrán castellano "mujer enferma, mujer eterna".

Algo muy importante a tener en cuenta en relación con la composición del retrato renacentista en aquella época, y mucho más del femenino, era el decorum, es decir, lo apropiado, lo pertinente. Por eso, los gestos, ademanes y expresiones para una dama respetable que iba a ser retratada estaban muy limitados: el rostro serio, el porte erguido, las manos descansando sobre los brazos de un sillón, sosteniendo un pañuelo, un abanico o permaneciendo unidas en señal de modestia; a este respecto es significativo que el Decor puellarum, un tratado de moral femenina publicado en Venecia en 1471, recomienda a las jóvenes, tanto si están caminando como paradas, mantener siempre la mano derecha sobre la izquierda a la altura del talle, algo que cumple escrupulosamente Lisa del Giocondo en su posado. La presencia del velo es otro de los atributos usuales en los retratos 
de esposas como signo de virtud y honestidad de la retratada, lo que unido en este caso a las palabras de amor y respeto que su esposo Francesco la dedica en el testamento redactado en 1537 , llegándola a calificar de mulier ingenua (35), o sea, sencilla, hablarían de una joven virtuosa, modesta, tímida, sobria en el vestir, poco dada a las joyas y de la que, por tanto, no es de extrañar que a Leonardo le costara bastante, según cuenta Vasari, conseguir que esbozase una sonrisa. Por cierto, la primera en toda la historia del retrato femenino. Una sonrisa que, amén de melancólica, enigmática, risueña, irónica, apasionada, compasiva, erótica, dulce, sarcástica, provocativa, vampírica, mundana, diabólica, tímida, ardiente, angelical y mil calificativos más, se ha definido, como hemos visto, de gravídica, desdentada, sifilítica, bruxista, etílica, hipoacúsica, distónica, oligofrénica y paralítica. Sonrisa que fue capaz de enamorar tanto al empresario Leon Mekusa (36) como para impulsarle a vender todos sus bienes en 1981, entrar como vigilante en el Louvre y así poder contemplarla todas las mañanas antes que nadie; capaz de excitar el ardor patriótico del italiano Vincenzo Peruggia (37), antiguo empleado del museo, empujándole en 1911 a robar el cuadro con la intención de reintegrarlo al patrimonio cultural italiano; o lo que es más dramático, capaz de inducir al suicidio del artista francés Luc Masperó (38), que en 1852 se lanzó al vacío desde la ventana del cuarto piso de su hotel parisino ante su incapacidad para descifrar la enigmática sonrisa, según nota manuscrita. Pues bien, resulta que semejante sonrisa de ambigüedad emocional o de melancolía risueña, como la ha definido el profesor Alonso-Fernández (39) y que recientemente ha servido como patrón de referencia en un estudio (40) sobre la percepción visual del estado emocional facial, en opinión de la neurofisióloga Margaret Livingstone (41) no existe. Se trataría sencillamente de la feliz coincidencia entre las peculiaridades anatomofisiológicas de la visión y la magistral técnica del $s f u$ mato desarrollada por Leonardo. De tal modo que cuando fijamos detenidamente nuestra mirada en la boca de la Gioconda tenemos la impresión de que su sonrisa se desvanece o incluso parece melancólica; por el contrario, si miramos la obra en su conjunto, se torna de nuevo risueña. La explicación, según la Dra. Livingstone, estaría en el procesamiento de la información visual y las características diferenciales entre la visión nítida o foveana y la visión periférica o de conjunto. Si a esto último añadimos, además, la transición uniforme y sin soluciones de continuidad entre luces y sombras conseguida por Leonardo con el sfumato, se reforzaría la percepción de la sonrisa al contemplar la obra en su conjunto. Es decir, la modelo permanece sonriente hasta que la miramos detenidamente a los labios, momento en el cual, como si de una superposición dinámica de dos estados, el risueño y el melancólico, con sonrisa o sin ella, fijáramos el último. Un fenómeno que a cualquier físico le recordaría uno de los principios básicos de la mecánica cuántica, el de Incertidumbre de Heisemberg. Y así lo refrenda el profesor Prvanovic, del Instituto de Física de Belgrado, con un artículo que lleva el significativo título de "Mona Lisa-ineffable smile of quantum mechanics" (42).

En resumen, y tomando en consideración toda la información proporcionada por unos procedimientos diagnósticos algo limitados, no sería aventurado afirmar que no existe una Mona Lisa. Simplemente, el logro más extraordinario conseguido por el gran Leonardo es que cada uno tengamos la nuestra.

\section{Bibliografía}

1. Gombrich EH. Historia del Arte. Madrid: Editorial Debate, 2003, p.300.

2. Keele KD. The genesis of Mona Lisa. J Hist Med Allied Sci 1959;14 (2):135-159.

3. Nulland SB. Leonardo da Vinci. New York: Penguin Books, 2000, p.80-81.

4. Cruz y Hermida J. La Gioconda vista por un médico. Málaga: Grupo Editorial 33, 2002, p.45.

5. Adour KK. Mona Lisa syndrome: solving the enigma of the Gioconda smile. Ann Otol Rhinol Laryngol 1989;98 (3):196-199.

6. Cruz y Hermida J. op.cit. p.60.

7. Cruz y Hermida J. op. Cit. p.62-63.

8. Borkowski JE. Mona Lisa: the enigma of the smile. J Forensic Sci 1992;3 (6):1706-1711.

9. Cruz y Hermida J. op. cit. p.48-49.

10. Freeth C. Ancient history of trips to the dentist. British Archaeology 1999;43:8-9.

11. Cruz y Hermida J. op. cit. p.73.

12. Lay-Son L. El retrato de Mona Lisa. Una visión neurológica. Rev Neurol 2003;36 (4):398.

13. Cruz y Hermida J. op. cit. p.37.

14. Cruz y Hermida J. op. cit. p.37-38

15. Dequeker J, Muls E, Leenders K. Xanthelasma and lipoma in Leonardo da Vinci's Mona Lisa. Isr Med Assoc J 2004;6 (8):505-506.

16. Cruz y Hermida J. op. cit. p.16.

17. Cruz y Hermida J. op. cit. p.46.

18. Cruz y Hermida J. op. cit. p.45.

19. Cruz y Hermida J. op. cit. p.43.

20. Lay-Son L. El retrato de Mona Lisa. Una visión neurológica. Rev Neurol 2003;36 (4):398.

21. Cruz y Hermida J. op. cit. p.63.

22. Cruz y Hermida J. op. cit. p.63.

23. Cruz y Hermida J. op. cit. p.64.

24. Cruz y Hermida J. op. cit. p.67.
25. Cruz y Hermida J. op. cit. p.65.

26. Viroli G. En: I Borgia. Milano: Electa, 2002, p.268.

27. Sassoon D. Becoming Mona Lisa: The making of a global icon. New York: Harcourt, 2001, p.29-30.

28. Vecce C. La Gualanda. ALV Journal 1999;III:51-71.

29. Laurelli F. Il ritratto di Monna Lisa Gherardini, detta "La Gioconda". Rivista Storica del Sannio 2000 ; 1 :268-303.

30. Tanaka H. Leonardo's Isabella d'Este: a new analysis of the Mona Lisa in the Luovre. Annuario 1976-77;13:23-34.

31. Freud S. Un recuerdo infantil de Leonardo da Vinci. Obras completas, vol. VIII. Madrid: Biblioteca Nueva, 1934, p.241-323.

32. Schwartz L. The Art Historian's Computer. Sci Am 1995;272 (4):106111.

33. Zöllner F. Leonardo's portrait of Mona Lisa del Giocondo. Gazette des Beaux Arts 1993;121:115-138.

34. Zöllner F. Leonardo's portrait of Mona Lisa del Giocondo. Gazette des Beaux Arts 1993;121:115-138.

35. Pallanti G. Monna Lisa. Mulier ingenua. Firenze: Edizioni Polistampa, 2004.

36. Sassoon D. op.cit. p.265-266.

37. Esterow M. The Art Stealers. New York: Macmillan Company, 1966. p.100-152.

38. Harris JA. Seeking Mona Lisa. Smithsonian 1999;30 (2):54-66.

39. Cruz y Hermida J. op. cit. p.11.

40. Kontsevich LL, Tyler CW. What makes Mona Lisa smile?. Vision Res 2004;44 (13):1493-1498.

41. Livingstone M. Is it warm? Is it real? or just low spatial frequency. Science 2000;290:1299.

42. Prvanovic S. Mona Lisa-Ineffable smile of quantum mechanics. arXiv:physics/0302089v1, 25 Feb 2003 [consultado 11-10-2005]: Disponible en: http://www.citebase.org/cgi-bin/citations?id=oai:arXiv.org:physics/0302089 